\title{
Influence of the measurement on the decay law: the bang-bang case
}

\author{
Francesco Giacosa ${ }^{1,2}$ and Giuseppe Pagliara ${ }^{3}$ \\ ${ }^{1}$ Institute of Physics, Jan Kochanowski University, 25-406 Kielce, Poland \\ ${ }^{2}$ Institute for Theoretical Physics, J. W. Goethe University, \\ Max-von-Laue-Str. 1, D-60438 Frankfurt am Main,Germany \\ ${ }^{3}$ Dip. di Fisica e Scienze della Terra dell'Università di Ferrara and \\ INFN Sez. di Ferrara, Via Saragat 1, I-44100 Ferrara, Italy
}

\begin{abstract}
After reviewing the description of an unstable state in the framework of nonrelativistic Quantum Mechanics (QM) and relativistic Quantum Field Theory (QFT), we consider the effect of pulsed, ideal measurements repeated at equal time intervals on the lifetime of an unstable system. In particular, we investigate the case in which the 'bare' survival probability is an exact exponential (a very good approximation in both QM and QFT), but the measurement apparatus can detect the decay products only in a certain energy range. We show that the Quantum Zeno Effect can occur in this framework as well.
\end{abstract}

\section{Introduction}

The study of decays is important in atomic, nuclear, and particle physics. Quite remarkably, although weak decays of nuclei (e.g. double- $\beta$ decays with lifetime $\sim 10^{21} \mathrm{y}$ ), and fast decays of hadrons (with lifetime $\sim 10^{-22} \mathrm{sec}$ ) are characterized by very different decay times, the basic phenomenon is the same: a coupling of an initial unstable state to a continuum of final states, which results in an irreversible quantum transition (infinite Poincare' time).

It is now well established both in Quantum Mechanics (QM) [1-3] and in Quantum Field Theory (QFT) $[4,5]$ that the survival probability $p(t)$ of an unstable state $|S\rangle$ is never exactly an exponential function: deviations at short as well as at long times take place. In the context of QM, these deviations have been verified experimentally at short-times in Ref. [6] and at long times in Ref. [7]. In particular, at short times the behavior $p(t) \simeq 1-t^{2} / \tau_{Z}^{2}$ occurs. In turn, the so-called Quantum Zeno Effect (QZE) takes place if repeated ideal measurements at time intervals $\tau \lesssim \tau_{Z}$ are performed (bang-bang measurements) [8-12]. The non-decay probability after $N$ measurements (i.e. at the time $t=N \tau$ ) is then given by

$$
\left.p(\tau)^{N} \simeq\left(1-t^{2} / \tau_{Z}^{2}\right)^{N} \simeq \exp \left[-\frac{t^{2}}{N \tau_{Z}^{2}}\right] \rightarrow 1 \text { for } N \rightarrow \infty \text { (i.e. } \tau \rightarrow 0, t \text { fixed }\right)
$$

Experimentally, the QZE was observed by inhibition of a Rabi oscillation between atomic energy levels in Ref. [13, 14] and, for a genuine unstable quantum state (tunneling), in Ref. [15]. 
Quite interestingly, as shown theoretically in Ref. [16] and experimentally verified in Ref. [17], the QZE takes place also for continuous measurements. In the latter case, a parameter, denoted as $\sigma$, is introduced to model the coupling of the decay products with the detector (a large value of $\sigma$ implies an intense coupling of the decay products with the detector). The time evolution is identical to Eq. (1) upon the identification $\tau=4 / \sigma$ (the so-called Schulman relation). In turn, in this limit the bang-bang and the continuous evolutions are indistinguishable.

Deviations from the exponential decay law are usually very small, and for this reason they could be measured only in engineered systems. Indeed, both in QM and in QFT the exponential decay is usually a very good approximation. There is however an intriguing possibility to obtain the QZE also in the case of a purely exponential decaying system: the use of an "imperfect detector", that is a detector which for instance can measure the final state only if the latter has an energy in a certain range (i.e. there is the possibility of an undetected decay). In Refs. [12, 18-20] this case has been studied theoretically by using models with a continuous coupling of the decay products with an imperfect detector. It has been recently re-investigated in Ref. [21] in which the case of bang-bang measurements has been also studied in detail. It has been found that the QZE occurs also for repeated pulsed bangbang measurements in which the collapse of the wave function takes place; moreover, the outcomes of bang-bang and continuous measurements are in general different (i.e., the Schulman relation does not hold), a property which has interesting implications for what concerns the interpretation of QM.

In these proceedings we first review the decay law both in QM and in QFT in Sec. 2, where we concentrate on the derivation of the exponential limit. Then, in Sec. 3 we study the effect of a series of ideal measurements which can detect the final state only in a certain frequency (or energy) band. Finally, we present our conclusions in Sec. 4.

\section{Description of an unstable state}

\subsection{QM: Lee Hamiltonian}

The Lee Hamiltonian describing the decay of an unstable state $|S\rangle$ into a final state $|k\rangle$ is given by $H=H_{0}+H_{1}$ with $[5,22,23]$ :

$$
H_{0}=M_{0}|S\rangle\left\langle S\left|+\int_{-\infty}^{+\infty} d k \omega(k)\right| k\right\rangle\langle k|, H_{1}=\int_{-\infty}^{+\infty} d k \frac{g f(k)}{\sqrt{2 \pi}}(|S\rangle\langle k|+\text { h.c. }) .
$$

The state $|k\rangle$ describes the decay products, enumerated by the continuous index $k$, which is assumed to vary between $-\infty$ and $+\infty$. Usually, $|k\rangle$ represents a two-particle state, emitted back-to-back. The survival probability amplitude of the state $|S\rangle$ reads

$$
a(t)=\left\langle S\left|e^{-i H t}\right| S\right\rangle=\frac{i}{2 \pi} \int_{-\infty}^{+\infty} d E\left\langle S\left|[E-H+i \varepsilon]^{-1}\right| S\right\rangle e^{-i E t},
$$

where in the r.h.s. the time evolution operator $e^{-i H t}$ has been (re)written as its operatorial Fourier transform $\frac{i}{2 \pi} \int_{-\infty}^{+\infty} d E[E-H+i \varepsilon]^{-1} e^{-i E t}$. The survival probability is obtained by squaring the amplitude: $p(t)=|a(t)|^{2}$. Using the equality $(E-H+i \varepsilon)^{-1}=\sum_{n=0}^{\infty}\left[\left(E-H_{0}+i \varepsilon\right)^{-1} H_{1}\right]^{n}\left(E-H_{0}+i \varepsilon\right)^{-1}$, the propagator of $|S\rangle$ is defined and calculated as

$$
G_{S}(E)=\left\langle S\left|\frac{1}{E-H+i \varepsilon}\right| S\right\rangle=\frac{1}{E-M_{0}+g^{2} \Sigma(E)+i \varepsilon}
$$

with the self-energy

$$
\Sigma(E)=-\int_{-\infty}^{+\infty} \frac{d k}{2 \pi} \frac{f^{2}(k)}{E-\omega(k)+i \varepsilon} .
$$


If $E$ falls within a certain support $I$, the imaginary part of $\Sigma(E)$ has the explicit expression

$$
\operatorname{Im} \Sigma(E)=\frac{1}{2} \frac{f^{2}\left(k_{E}\right)}{\left(\frac{d \omega}{d k}\right)_{k=k_{E}}}
$$

where $k_{E}$ is defined by $\omega\left(k_{E}\right)=E$. If $E$ lies outside $I$, the imaginary part simply vanishes. The (renormalized) nominal mass $M$ of the state $|S\rangle$ is defined as the solution of the equation:

$$
M-M_{0}+g^{2} \operatorname{Re} \Sigma(M)=0 .
$$

By expanding the real part of $G_{S}^{-1}(E)$ around $M$ we obtain the Breit-Wigner approximation of the propagator (by eliminating an inessential constant) as

$$
G_{S}^{\mathrm{BW}}(E)=\frac{1}{E-M+i \Gamma_{\mathrm{BW}} / 2}
$$

where the decay width $\Gamma_{\mathrm{BW}}$ is given by a generalization of the Fermi golden rule:

$$
\Gamma_{\mathrm{BW}}=\frac{g^{2}}{1+g^{2}\left(\frac{\partial \operatorname{Re} \Sigma(E)}{\partial E}\right)_{E=M}} \frac{f^{2}\left(k_{M}\right)}{\left(\frac{d \omega}{d k}\right)_{k=k_{M}}}
$$

and where $k_{M}$ is given by $\omega\left(k_{M}\right)=M$. Then, by using the approximation (8) the survival probability amplitude is given by $a(t)=\left\langle S\left|e^{-i H t}\right| S\right\rangle=e^{-i\left(M-i \Gamma_{\mathrm{BW}} / 2\right) t}$ and the survival probability takes the usual form $p(t)=|a(t)|^{2}=e^{-\Gamma_{\mathrm{BW}} t}$.

For illustrative purposes we present a simple model in which two simplifying assumptions for the functions $\omega(k)$ and $f(k)$ are made [24-26]: (i) $\omega(k)=k$. This choice implies that the energy is not bounded from below. Thus, it is obviously an -indeed a quite general- approximation, which is also needed to derive the usual exponential decay. (ii) $f(k)=\theta\left(M_{0}+\Lambda-k\right) \theta\left(k-\left(M_{0}-\Lambda\right)\right)$. In this way, the unstable state $|S\rangle$ couples in a limited window of energy to the final states of the type $|k\rangle$. Conditions (i) and (ii) define a toy model for the decay law in the general framework of Lee Hamiltonians. The self-energy $\Sigma(E)$ reads:

$$
\Sigma(E)=\frac{1}{2 \pi} \ln \left(\frac{E-M_{0}+\Lambda}{E-M_{0}-\Lambda}\right),
$$

whose real and imaginary parts are $\operatorname{Re} \Sigma(E)=\frac{1}{2 \pi} \ln \left|\frac{E-M_{0}+\Lambda}{E-M_{0}-\Lambda}\right|$ and $\operatorname{Im} \Sigma(E)=\frac{1}{2}$ for $M_{0}-\Lambda<E<$ $M_{0}+\Lambda, 0$ otherwise.

When $\Lambda$ is finite, deviations both at short and long times occur, see the explicit numerical results in [24-26]. However, in the limit $\Lambda \rightarrow \infty$ the cutoff model reduces exactly to the BW case and thus to the pure exponential decay. Namely, $\operatorname{Re} \Sigma(E)=0$ and $\operatorname{Im} \Sigma(E)=g^{2} / 2$ for each value of $E$ and the propagator reads $G_{S}(E)=[E-M+i \Gamma / 2]^{-1}$ with $M=M_{0}$ and $\Gamma=\Gamma_{\mathrm{BW}}=g^{2}$. The time-evolution operator applied to the unstable state $|S\rangle$ delivers the following result [23, 27-29]:

$$
\begin{aligned}
e^{-i H t}|S\rangle & =e^{-i\left(M_{0}-i \Gamma / 2\right) t}|S\rangle+\int_{-\infty}^{+\infty} d k b(k, t)|k\rangle \\
\text { with } b(k, t) & =\frac{g}{\sqrt{2 \pi}} \frac{e^{-i k t}-e^{-i\left(M_{0}-i \Gamma / 2\right) t}}{k-M_{0}+i \Gamma / 2} .
\end{aligned}
$$

The survival probability amplitude is $a(t)=\left\langle S\left|e^{-i H t}\right| S\right\rangle=e^{-i\left(M_{0}-i \Gamma / 2\right) t}$, from which $p(t)=e^{-\Gamma t}$. Notice that $e^{-i H t}|k\rangle=e^{-i k t}|k\rangle$ holds in the present case. 


\subsection{QFT: relativistic Lagrangian}

In the framework of relativistic QFT one obtains a picture similar to the QM case. In order to recall the main features we use the following Lagrangian with the scalar fields $S$ and $\varphi[4,5,30]$ :

$$
\mathcal{L}=\frac{1}{2}\left(\partial_{\mu} S\right)^{2}-\frac{1}{2} M_{0}^{2} S^{2}+\frac{1}{2}\left(\partial_{\mu} \varphi\right)^{2}-\frac{1}{2} m^{2} \varphi^{2}+g S \varphi^{2} .
$$

The decay process $S \rightarrow \varphi \varphi$ is analogous to the transition $|S\rangle \rightarrow|k\rangle$ of the previous subsection. The propagator of the particle $S$ is obtained upon Dyson resummation as

$$
G_{S}\left(p^{2}\right)=-i \int d^{4} y e^{-i p \cdot y}\langle 0|T[S(0) S(y)]| 0\rangle=\frac{1}{p^{2}-M_{0}^{2}+(\sqrt{2} g)^{2} \Sigma\left(p^{2}\right)+i \varepsilon}
$$

where $T$ is the chronological product, $|0\rangle$ the (non-perturbative) vacuum state, and $\Sigma\left(p^{2}\right)$ the selfenergy . The form of Eq. (13) is analogous to Eq. (4). in the one-loop approximation (which is usually a very good one in this context, see the recent Ref. [31].) $\Sigma\left(p^{2}\right)$ reads [5, 30]:

$$
\Sigma\left(x^{2}\right)=\int \frac{d^{3} q}{(2 \pi)^{3}} \frac{\tilde{\phi}^{2}(\mathbf{q})}{\sqrt{\mathbf{q}^{2}+m^{2}}\left(4\left(\mathbf{q}^{2}+m^{2}\right)-x^{2}-i \varepsilon\right)} .
$$

which has a form very similar to Eq. (5) (for a more detailed comparison of QM and QFT, see Ref. [5]). The choice of the vertex-function $\tilde{\phi}(\mathbf{q})$ depends on the model and on the physics that one wants to study [30, 32]. For illustrative purposes, the cutoff choice $\tilde{\phi}(\mathbf{q})=\theta\left(\mathbf{q}^{2}-\Lambda^{2}\right)$ is useful and represents the analogous expression of the 'cutoff model' of the QM case.

The renormalized mass $M$ of the particle $S$ is defined as the zero of the real part of $G_{S}\left(p^{2}\right)^{-1}$ : $M^{2}-M_{0}^{2}+\operatorname{Re} \Pi\left(M^{2}\right)=0$. Usually $\operatorname{Re} \Pi\left(M^{2}\right)>0$, thus $M$ is smaller than $M_{0}$. The tree-level decay width of the process $S \rightarrow \varphi \varphi$ reads:

$$
\Gamma=\Gamma^{\mathrm{tl}}(x=M), \Gamma^{\mathrm{tl}}(x)=\frac{\sqrt{\frac{x^{2}}{4}-m^{2}}}{8 \pi x^{2}}(\sqrt{2} g)^{2} \theta(x-2 m) .
$$

In order to obtain the BW expression of the propagator two steps are necessary. First, one simplify $G_{S}$ via the so-called relativistic BW approximation as

$$
G_{S} \simeq \frac{N_{\mathrm{BWrel}}}{x^{2}-M^{2}+i M \Gamma}
$$

where $N_{\mathrm{BWrel}}$ is a renormalization constant. Then, one obtains the non-relativistic case in the following way:

$$
G_{S} \simeq \frac{N_{\mathrm{BWrel}}}{(x-M)(2 M)+i M \Gamma} \simeq \frac{N_{\mathrm{BW}}}{x-M+i \Gamma / 2} .
$$

Thus, we obtain from the relativistic framework also the nonrelativistic BW propagator as a special limit. It is indeed remarkable that this limit works so well also for strong decaying particles, as for instance for the $\rho$ meson.

\section{Pulsed measurements with an 'imperfect' detector}

Let us now consider the case in which the intrinsic decay is exactly an exponential (i.e., it is assumed that the small deviations from the exponential decay are completely negligible for our purposes.) We 
aim to show which is the effect of a series of imperfect measurements at time intervals $\tau, 2 \tau, \ldots, t=n \tau$. The discussion is taken from Ref. [21], to which we add some technical details of the derivation of the main formula of that paper.

In the exponential limit Eq. (11) holds. Without loss of generality, we set $M_{0}=0$. The state of the system at the instant $t>0$ is given by $|\Psi(t)\rangle=e^{-i H t}|S\rangle$ (valid up to the the first measurement). Then, we assume to perform a measurement at the instant $\tau$ and that the detector $D$ can only detect the final state $|k\rangle$ if $-\lambda \leq k \leq \lambda$. This means that the probability to "hear" the click of the detector is given by:

$$
p_{\text {click }}(\tau)=w_{\lambda}(\tau)=\int_{-\lambda}^{\lambda}|b(k, \tau)|^{2} d k
$$

Conversely, the no-click probability after the first measurement is $p_{\text {no-click }}(\tau)=1-w_{\lambda}(\tau)$. (Note, for $\lambda \rightarrow \infty$ the probability that the detector makes click for a single measurement at the instant $\tau$ is $w_{\infty}(\tau)=\int_{-\infty}^{+\infty} d k|b(k, \tau)|^{2}=1-p(\tau)=1-e^{-\Gamma t}$, as expected.) Then, if the detection has not taken place, the state of the system at the instant of time $\tau^{+}$(just after the first measurement) is given by

$$
\left|\Psi\left(\tau^{+}\right)\right\rangle=N\left[e^{-\tau \Gamma / 2}|S\rangle+\int_{R} d k b(k, \tau)|k\rangle\right]
$$

where $R=(-\infty,-\lambda) \cup(\lambda, \infty)$ and $N=1 / \sqrt{p_{\text {no-click }}(\tau)}$ is a normalization constant arising after that the wave function has collapsed (the band $W=(-\lambda, \lambda)$ has disappeared from the linear superposition because no click has occurred). Further unitary time-evolution implies that for $t>\tau$ (valid up to the second measurement):

$$
\left|\Psi\left(t=\tau+t^{\prime}\right)\right\rangle=N\left[e^{-\tau \Gamma / 2}\left(e^{-t^{\prime} \Gamma / 2}|S\rangle+\int_{-\infty}^{+\infty} d k b\left(k, t^{\prime}\right)|k\rangle\right)+\int_{R} d k b(k, \tau) e^{-i k t^{\prime}}|k\rangle\right] .
$$

Note, the normalization of the state for $t>\tau$ implies that

$$
|N|^{2}\left[p(\tau) p\left(t^{\prime}\right)+p(\tau) w_{\lambda}\left(t^{\prime}\right)+X\left(t^{\prime}\right)\right]=1 \text { valid } \forall t^{\prime}
$$

with $X\left(t^{\prime}\right)=\left|\int_{R} d k\left[b\left(k, t^{\prime}\right) e^{-i k t^{\prime}}+e^{-\tau \Gamma / 2} b\left(k, t^{\prime}\right)\right]\right|^{2}$. Now, we assume to make a second measurement at the time $t=2 \tau$. The probability to hear a click at the time $2 \tau$ is given by $p_{\text {click }}(2 \tau)=$ $p_{\text {no-click }}(\tau)|N|^{2} p(\tau) w_{\lambda}(\tau)=p(t) w_{\lambda}(\tau)$. Conversely, if no click takes place also at $t=2 \tau$, just slightly after it we have:

$$
\left|\Psi\left(t=2 \tau^{+}\right)\right\rangle=\tilde{N}\left[e^{-\tau \Gamma / 2}\left(e^{-\tau \Gamma / 2}|S\rangle+\int_{R} d k b(k, \tau)|k\rangle\right)+\int_{R} d k b(k, \tau) e^{-i k \tau}|k\rangle\right]
$$

where $\tilde{N}$ is a new normalization constant after the second collapse. By using Eq. (20) one obtains:

$$
|\tilde{N}|^{2}\left[p(\tau)^{2}+X(\tau)\right]=1 \rightarrow|\tilde{N}|^{2}\left[\frac{1}{|N|^{2}}-p(\tau) w_{\lambda}(\tau)\right]=1
$$

from which:

$$
|\tilde{N}|^{2}=\frac{1}{1-w_{\lambda}(\tau)-p(\tau) w_{\lambda}(\tau)} .
$$

Then, one can repeat the procedure as often as desired. The next step is a measurement at the time $t=3 \tau$, and so on. The outcome is that the probability to hear a click exactly at the $n$-th measurement is given by:

$$
p_{\text {click }}(n \tau)=p^{n-1}(\tau) w_{\lambda}(\tau) \text {. }
$$




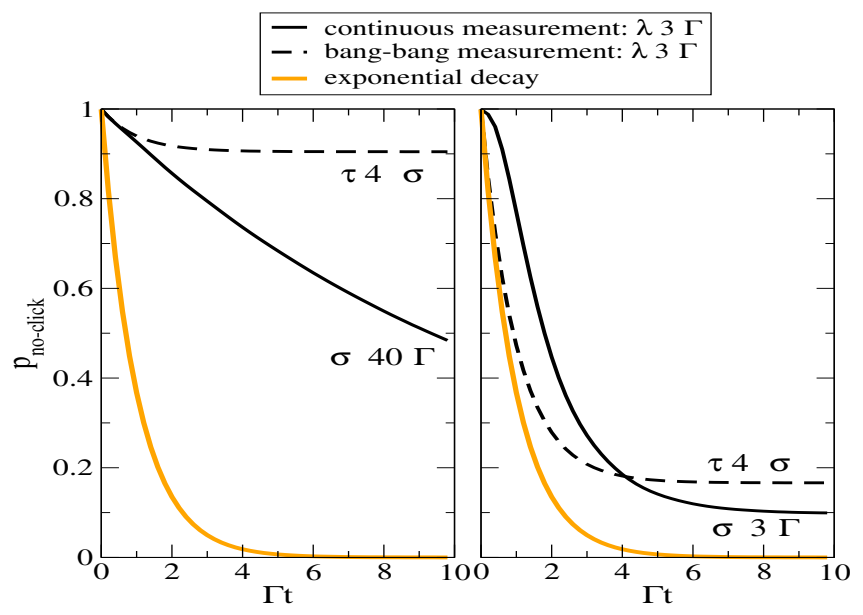

Figure 1. Temporal evolution of the no-click probabilities in the case of exponential decay, continuous and pulsed measurements for two values of the efficiency of detection $\sigma$ (from [21]).

This formula is intuitive, as it expresses the probability to hear click at the $n$-th measurement as the joint probability that the state $|S\rangle$ is still present at the time $(n-1) \tau$ and that the decay takes place exactly in the very last step. From Eq. (24) we calculate the no-click probability up to the instant $t=n \tau$ as:

$$
\begin{aligned}
p_{\text {no-cklick }}(t & =n \tau)=1-\sum_{k=1}^{n} p_{\text {click }}(k \tau)=1-\sum_{k=1}^{n} p^{k-1}(\tau) w_{\lambda}(\tau) \\
& =1-w_{\lambda}(\tau) \frac{1-p(\tau)^{n}}{1-p(\tau)}=1-w_{\lambda}(\tau) \frac{1-e^{-\Gamma t}}{1-e^{-\Gamma \tau}} .
\end{aligned}
$$

This is indeed one of the main results of Ref. [21], which we have here derived with more details.

In Fig.1, we display two numerical examples of Eq. (25) (dashed lines) corresponding to two values of $\tau$ in comparison with the exponential survival probability (orange lines). Notice that the evolution of the system is strongly slowed down due to the repeated measurements: after a time period of 5 times the lifetime of the unstable system, the no-click probability is still of the order of 90\% in the left panel and 20\% in the left panel. This is the usual QZE which indeed takes place also in presence of exponentially decaying systems but which are "observed" by imperfect detectors. Another interesting feature is that the no-click probability saturates to a finite value at late times. In the figure we also show results for continuous measurements (solid lines) with $\sigma$ related to $\tau$ by the Schulman relation [16]. Contrary to the case of perfect detectors, in the case of imperfect ones the results for continuous and pulsed measurements differ from each other. As discussed in Ref. [21], the qualitative difference between these two types of measurements could in principle be exploited for clarifying whether the collapse of the wave function, which has been explicitly constructed previously for deriving equation (25), is a real physical process playing a fundamental role for the QZE.

\section{Discussions and conclusions}

In this work we have first reviewed the emergence of the Breit-Wigner limit, and conversely of the exponential decay, in both QM and QFT. To this purpose we have studied the propagators in QM and 
QFT and presented the analogies between them. Quite interestingly, the exponential limit, although never exact, works often very well in both cases.

As a next step we concentrated our attention to the case in which the decay law is an exact exponential but the detector can detect the decay products only in a certain range of energy (it is in a sense an "imperfect detector" since in some cases the decayed particles pass undetected). In this case, even if the intrinsic decay is treated as an exact exponential function, the quantum Zeno effect can be induced by frequent measurements of the ideal type (collapse of the wave function after each measurement). We have shown how the probability of not hearing a click (which is not equal to the survival probability because the detector has not a $100 \%$ efficiency) is derived and some numerical example has been also provided.

In the future, one should investigate further a detector modelled by a series of ideal but imperfect measurements by taking into account that they do not take place at equal time intervals. Further comparison with the alternative view of a continuous measurement is interesting, see Ref. [21] and references therein. This comparison has also important implications for fundaments of QM, i.e. if the collapse of the wave function is a real physical process as proposed in Refs. [33-37], or not, see e.g. $[38,39]$.

\section{References}

[1] L. A. Khalfin, 1957 Zh. Eksp. Teor. Fiz. 33 1371. (Engl. trans. Sov. Phys. JETP 6 1053).

[2] L. Fonda, G. C. Ghirardi and A. Rimini, Rept. Prog. Phys. 41 (1978) 587.

[3] C. A. Nicolaides and T. Mercouris, Journal of Physics B 29, Issue 6, pp. 1151-1167 (1996). K. Urbanowski, Central European Journal of Physics 7, Issue 4, pp.696-703. E. Torrontegui, J. G. Muga, J. Martorell, J, and D. W. L. Sprung, Phys. Rev. A 80, Issue 1, 012703 (2009).

[4] F. Giacosa, G. Pagliara, Mod. Phys. Lett. A26 (2011) 2247-2259. [arXiv:1005.4817 [hep-ph]]. G. Pagliara, F. Giacosa, Acta Phys. Polon. Supp. 4 (2011) 753-758. [arXiv:1108.2782 [hep-ph]]. F. Giacosa and G. Pagliara, Phys. Rev. D 88 (2013) 025010 [arXiv:1210.4192 [hep-ph]].

[5] F. Giacosa, Found. Phys. 42 (2012) 1262 [arXiv:1110.5923 [nucl-th]].

[6] S. R. Wilkinson, C. F. Bharucha, M. C. Fischer, K. W. Madison, P. R. Morrow, Q. Niu, B. Sundaram, M. G. Raizen, Nature 387, 575 (1997).

[7] C. Rothe, S. I. Hintschich, A. P. Monkman, Phys. Rev. Lett. 96 (2006)163601.

[8] A. Degasperis, L. Fonda and G. C. Ghirardi, Nuovo Cim. A 21 (1973) 471.

[9] B. Misra and E. C. G. Sudarshan, J. Math. Phys. 18 (1977) 756.

[10] P. Facchi, H. Nakazato, S. Pascazio Phys. Rev. Lett. 86 (2001) 2699-2703. P. Facchi and S. Pascazio, Phys. Lett. A 241 (1998) 139 [arXiv:quant-ph/9905017].

[11] A. G. Kofman and G. Kurizki, Nature (London) 405, 546 (2000); A. P. Balachandran and S. M. Roy, Phys. Rev. Lett. 84, 4019 (2000).

[12] K. Koshino and A. Shimizu, Phys. Rept. 412 (2005) 191 [quant-ph/0411145].

[13] W. M. Itano, D. J. Heinzen, J. J. Bollinger and D. J. Wineland, Phys. Rev. A 41 (1990) 2295.

[14] Chr. Balzer et al, Optics Communications, Volume 211, Issue 1-6, p. 235-241 (2002).

[15] M. C. Fischer, B. Gutiérrez-Medina and M. G. Raizen, Phys. Rev. Lett. 87, 040402 (2001).

[16] L.S. Schulman, Phys.Rev. A 57 1509n (1998).

[17] E. W. Streed et al, Phys. Rev. Lett. 97, Issue 26, 260402 (2006).

[18] K. Koshino and A. Shimizu, Phys. Rev. Lett. 92 (2004) 030401.

[19] K. Koshino, Phys. Rev. A 71034104 (2005). K. Koshino, Phys. Rev. Lett. 93, nr 3 (2004). H. Goto and I. Ichimura, Phys. Rev. A 78, 044102 (2008). 
[20] P. Facchi and S. Pascazio, Fortschritte der Physik 49, 941 (2001); P. Facchi and S. Pascazio, Time's arrows, quantum measurements and superluminal behavior, edited by D. Mugnai, R. Ranfagni and L.S. Schulman (CNR, Rome, 2001) p. 139, arXiv:quant-ph/0101044.

[21] F. Giacosa and G. Pagliara, to appear in Phys. Rev. A, [arXiv:1405.6882 [quant-ph]].

[22] T. D. Lee, Phys. Rev. 95 (1954) 1329-1334. C. B. Chiu, E. C. G. Sudarshan and G. Bhamathi, Phys. Rev. D 46 (1992) 3508.

[23] F. Giacosa, Phys. Rev. A 88, 052131 (2013) [arXiv:1305.4467 [quant-ph]].

[24] F. Giacosa, arXiv:1312.3315 [quant-ph].

[25] F. Giacosa, G. Pagliara , Quant. Matt. 2 (2013) 54 [arXiv:1110.1669 [nucl-th]]. F. Giacosa, G. Pagliara, PoS BORMIO 2012 (2012) 028 [arXiv:1204.1896 [nucl-th]].

[26] T. Wolkanowski, arXiv:1303.4657 [hep-ph].

[27] V. Weisskopf and E. P. Wigner, Z. Phys. 63 (1930) 54. V. Weisskopf and E. Wigner, Z. Phys. 65 (1930) 18. G. Breit, Handbuch der Physik 41, 1 (1959).

[28] M. O. Scully and M. S. Zubairy (1997), Quantum optics, Cambridge UK: Cambridge University Press.

[29] P. Facchi and S. Pascazio, Phys. Rev. A 62, 023804 (2000) [arXiv:quant-ph/9909043 ]. P. R. Berman and G. W. Ford, Phys. Rev. A 82, Issue 2, 023818. A. G Kofman, G. Kurizki, and B. Sherman, Journal of Modern Optics, vol. 41, Issue 2, p.353-384 (1994).

[30] F. Giacosa, G. Pagliara, Phys. Rev. C76 (2007) 065204. [arXiv:0707.3594 [hep-ph]]. F. Giacosa and T. Wolkanowski, Mod. Phys. Lett. A 27 (2012) 1250229 arXiv:1209.2332 [hep-ph]]. T. Wolkanowski and F. Giacosa, Acta Phys. Polon. Supp. 6 (2013) 893 [arXiv:1305.2603 [hep$\mathrm{ph}]]$.

[31] J. Schneitzer, T. Wolkanowski and F. Giacosa, Nucl. Phys. B 888 (2014) 287 [arXiv:1407.7414 [hep-ph]].

[32] N. N. Achasov and A. V. Kiselev, Phys. Rev. D 70 (2004) 111901 [hep-ph/0405128]. A. Faessler, T. Gutsche, M. A. Ivanov, V. E. Lyubovitskij and P. Wang, Phys. Rev. D 68 (2003) 014011 [hep-ph/0304031]. F. Giacosa, T. Gutsche and A. Faessler, Phys. Rev. C 71 (2005) 025202 [hepph/0408085]. F. Giacosa and G. Pagliara, Nucl. Phys. A 812 (2008) 125 [arXiv:0804.1572 [hep$\mathrm{ph}]]$.

[33] A. Bassi and G. C. Ghirardi, Phys. Rept. 379 (2003) 257 [quant-ph/0302164].

[34] A. Bassi, K. Lochan, S. Satin, T. P. Singh and H. Ulbricht, Rev. Mod. Phys. 85 (2013) 471 [arXiv:1204.4325 [quant-ph]].

[35] G. C. Ghirardi, O. Nicrosini, A. Rimini and T. Weber, Nuovo Cim. B 102 (1988) 383.

[36] L. Diosi, J. Phys. A 21 (1988) 2885. R. Penrose, Gen. Rel. Grav. 28 (1996) 581.

[37] Francesco Giacosa, [arXiv:1406.2344 [quant-ph]], to appear in 'Quanta'.

[38] H. Everett, Rev. Mod. Phys. 29 (1957) 454.

[39] D. Deutsch, quant-ph/9906015. S. Saunders, Proc.Royal Society A460 67-68 (2004). D. Wallace, Studies in the History and Philosophy of Modern Physics 34, 415-442 (2003). M. Schlosshauer, Rev. Mod. Phys. 76 (2004) 1267 [quant-ph/0312059]. M. Namiki and S. Pascazio, Phys. Rev. A 44 (1991) 39. 